\title{
INTERNAL STABILIZATION OF MAXWELL'S EQUATIONS IN HETEROGENEOUS MEDIA
}

\author{
SERGE NICAISE AND CRISTINA PIGNOTTI
}

Received 18 April 2004

We consider the internal stabilization of Maxwell's equations with Ohm's law with space variable coefficients in a bounded region with a smooth boundary. Our result is mainly based on an observability estimate, obtained in some particular cases by the multiplier method, a duality argument and a weakening of norm argument, and arguments used in internal stabilization of scalar wave equations.

\section{Introduction}

Let $\Omega$ be an open bounded domain in $\mathbb{R}^{3}$ with a boundary $\Gamma$ of class $C^{2}$. For the sake of simplicity we further assume that $\Omega$ is simply connected and that $\Gamma$ is connected.

In this paper we study the stabilization of Maxwell's equations with Ohm's law:

$$
\begin{gathered}
D^{\prime}-\operatorname{curl}(\mu B)+\sigma D=0 \quad \text { in } \Omega \times(0,+\infty), \\
B^{\prime}+\operatorname{curl}(\lambda D)=0 \quad \text { in } \Omega \times(0,+\infty), \\
\operatorname{div} B=0 \quad \text { in } \Omega \times(0,+\infty), \\
D(0)=D_{0}, \quad B(0)=B_{0} \quad \text { in } \Omega, \\
D \times v=0, \quad B \cdot v=0 \quad \text { on } \Gamma \times(0,+\infty),
\end{gathered}
$$

where $D, B$ are three-dimensional vector-valued functions of $t, x=\left(x_{1}, x_{2}, x_{3}\right) ; \mu=\mu(x)$, $\lambda=\lambda(x), \sigma=\sigma(x)$ are scalar functions in $C^{1}(\bar{\Omega})$ such that $\sigma(x) \geq 0$ and $\lambda$ and $\mu$ are uniformly bounded from below by a positive constant, that is,

$$
\lambda(x) \geq \lambda_{0}>0, \quad \mu(x) \geq \mu_{0}>0, \quad \forall x \in \bar{\Omega} .
$$

$D_{0}, B_{0}$ are the initial data in a suitable space and $\nu$ denotes the outward unit normal vector to $\Gamma$. We further assume that $\sigma$ satisfies

$$
\sigma(x) \geq \sigma_{0}>0, \quad \forall x \in \omega
$$

for some non empty open subset $\omega$ of $\Omega$. 
In that paper we will give sufficient conditions on $\lambda, \mu$ and $\omega$ which guarantee the exponential decay of the energy

$$
\mathscr{E}(t):=\frac{1}{2} \int_{\Omega}\left(\lambda(x)|D(x, t)|^{2}+\mu(x)|B(x, t)|^{2}\right) d x
$$

of our system.

The exact boundary controllability and stabilization of Maxwell's equations have been studied by many authors $[4,6,7,8,10,13,15,17,18,19,21]$ and are usually based on an observability estimate obtained by different methods like the multiplier method, microlocal analysis, the frequency domain method. A similar strategy leads to the internal controllability of Maxwell's equations, see for instance [17, 18, 22, 23].

But to our knowledge the internal stabilization of Maxwell's equations with Ohm's law is only considered for constant coefficients $\lambda$ and $\mu$ [17]. Therefore our goal is to consider the internal stabilization of Maxwell's equations with Ohm's law for space variable coefficients $\lambda$ and $\mu$. We then give sufficient conditions guaranteeing the exponential decay of the energy. Our method actually combines arguments used in the stabilization of scalar wave equation with locally distributed (internal) damping [24] with the use of an internal observability estimate for the standard Maxwell equations obtained for constant coefficients by Phung [17] using microlocal analysis and extended here to some subsets $\omega$ of $\Omega$ and space variable coefficients. This observability estimate is obtained using a vectorial multiplier method (see [11] in the scalar case and [22] for constant coefficients), a duality argument from $[1,12]$ and a weakening of norm argument (as in [11] in the scalar case).

The schedule of the paper is the following one: Well-posedness of the problem is analysed in Section 2 under appropriate conditions on $\Omega, \lambda$, $\mu$ and $\sigma$ using semigroup theory. Section 3 is devoted to the proof of the observability estimate when $\omega$ is a (small) neighbourhood of the boundary. Finally we conclude in Section 4 by the exponential stability of our system.

\section{Well-posedness of the problem}

Introduce the Hilbert spaces

$$
\begin{gathered}
\hat{J}(\Omega):=\left\{B \in L^{2}(\Omega)^{3}: \operatorname{div} B=0 \text { in } \Omega ; B \cdot \nu=0 \text { on } \Gamma\right\}, \\
H:=L^{2}(\Omega)^{3} \times \hat{J}(\Omega),
\end{gathered}
$$

equipped with the inner product

$$
\left(\left(\begin{array}{l}
D \\
B
\end{array}\right),\left(\begin{array}{l}
D_{1} \\
B_{1}
\end{array}\right)\right)_{H}=\int_{\Omega}\left\{\lambda D D_{1}+\mu B B_{1}\right\} d x .
$$

Now define the operator $A$ as follows:

$$
D(A)=H_{0}(\operatorname{curl}, \Omega) \times\left(\hat{J}(\Omega) \cap H^{1}(\Omega)^{3}\right),
$$


where, as usual,

$$
H_{0}(\operatorname{curl}, \Omega)=\left\{D \in L^{2}(\Omega)^{3}: \operatorname{curl} D \in L^{2}(\Omega)^{3}, D \times \nu=0 \text { on } \Gamma\right\} .
$$

For any $\left(\begin{array}{l}D \\ B\end{array}\right)$ in $D(A)$ we take

$$
A\left(\begin{array}{l}
D \\
B
\end{array}\right)=\left(\begin{array}{c}
\operatorname{curl}(\mu B)-\sigma D \\
-\operatorname{curl}(\lambda D)
\end{array}\right)
$$

We then see that formally problem (1.1) to (1.5) is equivalent to

$$
\begin{gathered}
\frac{\partial \Phi}{\partial t}=A \Phi, \\
\Phi(0)=\Phi_{0},
\end{gathered}
$$

when $\Phi=\left(\begin{array}{c}D \\ B\end{array}\right)$ and $\Phi_{0}=\left(\begin{array}{c}D_{0} \\ B_{0}\end{array}\right)$.

We will prove that this problem (2.6) has a unique solution using Lumer-Phillips' theorem [16] by showing the following lemma.

Lemma 2.1. A is a maximal dissipative operator.

Proof. We start with the dissipativeness of $A$, in other words we need to show that

$$
\mathfrak{R}(A \Phi, \Phi)_{H} \leq 0, \quad \forall \Phi \in D(A)
$$

With the above notation we have

$$
(A \Phi, \Phi)_{H}=\int_{\Omega}\{\lambda(\operatorname{curl}(\mu B)-\sigma D) \cdot D-\mu \operatorname{curl}(\lambda D) B\} d x .
$$

By Green's formula and the boundary condition $D \times \nu=0$ on $\Gamma$, we get

$$
(A \Phi, \Phi)_{H}=-\int_{\Omega} \lambda \sigma|D|^{2} d x \leq 0
$$

Let us now pass to the maximality. For that purpose it suffices to show that for all $\left(\begin{array}{l}f \\ g\end{array}\right)$ in $H$, there exists a unique $\left(\begin{array}{l}D \\ B\end{array}\right)$ in $D(A)$ such that

$$
(I-A)\left(\begin{array}{l}
D \\
B
\end{array}\right)=\left(\begin{array}{l}
f \\
g
\end{array}\right) .
$$

Equivalently, we have

$$
\begin{gathered}
B=g-\operatorname{curl}(\lambda D), \\
D+\operatorname{curl}(\mu \operatorname{curl}(\lambda D))+\sigma D=f+\operatorname{curl}(\mu g) .
\end{gathered}
$$


This last problem has a unique solution $D$ in $H_{0}(\operatorname{curl}, \Omega)$ because its variational formulation is

$$
\begin{aligned}
& \int_{\Omega}\{\mu \operatorname{curl}(\lambda D) \cdot \operatorname{curl}(\lambda w)+\lambda(1+\sigma) D \cdot w\} d x \\
& =\int_{\Omega}\{\lambda f \cdot w+\mu g \cdot \operatorname{curl}(\lambda w)\} d x, \quad \forall w \in H_{0}(\operatorname{curl}, \Omega) .
\end{aligned}
$$

This problem has a unique solution by the Lax-Milgram lemma because the bilinear form defined as the left-hand side is coercive on $H_{0}(\operatorname{curl}, \Omega)$ because $\lambda(1+\sigma) \geq \lambda_{0}$.

It then remains to show that $B$ given by $(2.11)$ belongs to $\hat{J}(\Omega) \cap H^{1}(\Omega)^{3}$. Indeed by (2.11), we see that

$$
\operatorname{curl}(\mu B)=(1+\sigma) D-f
$$

which shows that $\operatorname{curl} B \in L^{2}(\Omega)^{3}$. On the other hand $\operatorname{div} B=\operatorname{div} g=0$ since $g$ belongs to $\hat{J}(\Omega)$. Finally $B \cdot v=0$ on $\Gamma$ because the boundary condition $\lambda D \times \nu=0$ on $\Gamma$ implies that $\operatorname{curl}(\lambda D) \cdot \nu=0$ on $\Gamma$ and because $g \in \hat{J}(\Omega)$. Altogether we have that $B \in H_{T}(\operatorname{curl}, \operatorname{div}, \Omega)$, where

$$
\begin{aligned}
H_{T}(\operatorname{curl}, \operatorname{div}, \Omega):=\left\{B \in L^{2}(\Omega)^{3}: \operatorname{curl} B \in L^{2}(\Omega)^{3},\right. \\
\\
\left.\operatorname{div} B \in L^{2}(\Omega) ; B \cdot \nu=0 \text { on } \Gamma\right\} .
\end{aligned}
$$

Since the boundary $\Gamma$ is supposed to be smooth we have the continuous embedding $H_{T}(\operatorname{curl}, \operatorname{div}, \Omega) \hookrightarrow\left(H^{1}(\Omega)\right)^{3}$ (see, e.g., [5, Section I.3.4]), which leads to the requested regularity on $B$.

Since it is well-known that $D(A)$ is dense in $H$ (see [9, Section 7] or [10]), by LumerPhillips' theorem (see, e.g., [16, Theorem I.4.3]), we conclude that $A$ generates a $C_{0}$ semigroup of contraction $T(t)$. Therefore we have the following existence result.

Theorem 2.2. For all $\Phi_{0} \in H$, the problem (2.6) has a weak solution $\Phi \in C([0, \infty), H)$ given by $\Phi=T(t) \Phi_{0}$. If moreover $\Phi_{0} \in D(A)$, the problem (2.6) has a strong solution $\Phi \in$ $C([0, \infty), D(A)) \cap C^{1}([0, \infty), H)$.

For our further use we also need the next result.

Theorem 2.3. Fix $T>0$. Then for all $f \in L^{2}\left(0, T ; L^{2}(\Omega)^{3}\right)$, the problem

$$
\begin{gathered}
D^{\prime}-\operatorname{curl}(\mu B)=f \quad \text { in } Q_{T}:=\Omega \times(0, T), \\
B^{\prime}+\operatorname{curl}(\lambda D)=0 \quad \text { in } Q_{T}, \\
\operatorname{div} B=0 \quad \text { in } Q_{T}, \\
D(0)=0, \quad B(0)=0 \quad \text { in } \Omega, \\
D \times v=0, \quad B \cdot v=0 \quad \text { on } \Sigma_{T}:=\Gamma \times(0, T),
\end{gathered}
$$


has a unique mild solution $\left(\begin{array}{l}D \\ B\end{array}\right) \in C([0, T), H)$ which satisfies the estimate

$$
\int_{Q_{T}}\left\{|D(x, t)|^{2}+|B(x, t)|^{2}\right\} d x d t \leq C T^{2} \int_{Q_{T}}|f(x, t)|^{2} d x d t,
$$

for some positive constant $C$ depending on $\lambda$ and $\mu$.

Proof. Denoting by $A_{0}$ the above operator $A$ corresponding to $\sigma=0$, the above problem (2.16) to (2.20) is equivalent to

$$
\begin{gathered}
\frac{\partial \Phi}{\partial t}=A_{0} \Phi+F, \\
\Phi(0)=0,
\end{gathered}
$$

when $\Phi=\left(\begin{array}{l}D \\ B\end{array}\right)$ and $F=\left(\begin{array}{l}f \\ 0\end{array}\right)$.

As $A_{0}$ generates a $C_{0}$-semigroup of contraction $T_{0}(t)$, problem (2.22) has a unique mild solution $\Phi \in C([0, \infty), H)$ given by (see [16, Section 4.4.2])

$$
\Phi(t)=\int_{0}^{t} T_{0}(t-s) F(s) d s
$$

This identity implies that

$$
\|\Phi(t)\|_{H} \leq \int_{0}^{t}\|F(s)\|_{H} d s \leq \int_{0}^{t}\left(\int_{\Omega} \lambda(x)|f(x, s)|^{2} d x\right)^{1 / 2} d s .
$$

We conclude by integrating the square of this estimate in $t \in(0, T)$, using CauchySchwarz's inequality and taking into account the assumption (1.6).

We end this section by showing that the energy of our system is decreasing.

Lemma 2.4. Let $\left(D_{0}, B_{0}\right)$ be an initial pair in $H$ and let $(D, B)$ be the solution of the system (1.1), (1.2), (1.3), (1.4), and (1.5). Then the derivative of the energy (defined by (1.8)) is

$$
\mathscr{E}^{\prime}(t)=-\int_{\Omega} \lambda \sigma|D|^{2} d x \leq 0, \quad \forall t>0
$$

Proof. Deriving (1.8) we obtain

$$
\mathscr{E}^{\prime}=\int_{\Omega}\left\{\lambda D \cdot D^{\prime}+\mu B \cdot B^{\prime}\right\} d x
$$

then, by (1.1) and (1.2),

$$
\mathscr{E}^{\prime}=\int_{\Omega}\{\lambda D \cdot(\operatorname{curl} \mu B-\sigma D)-\mu B \cdot \operatorname{curl} \lambda D\} d x .
$$

We conclude by integrating by parts in the first term of this right-hand side and using the boundary condition (1.5).

From this lemma we directly conclude that the energy is non-increasing. 
Corollary 2.5. Let $\left(D_{0}, B_{0}\right)$ be an initial pair in $H$ and let $(D, B)$ be the solution of the system (1.1), (1.2), (1.3), (1.4), and (1.5). Then, for all $0 \leq S<T<+\infty$, we have

$$
\mathscr{E}(S)-\mathscr{E}(T)=\int_{S}^{T} \int_{\Omega} \lambda \sigma|D|^{2} d x \geq 0
$$

\section{An observability estimate}

Let us consider the solution $\left(D_{h}, B_{h}\right)$ of the standard Maxwell system:

$$
\begin{gathered}
D_{h}^{\prime}-\operatorname{curl}\left(\mu B_{h}\right)=0 \quad \text { in } \Omega \times(0,+\infty), \\
B_{h}^{\prime}+\operatorname{curl}\left(\lambda D_{h}\right)=0 \quad \text { in } \Omega \times(0,+\infty), \\
\operatorname{div} D_{h}=\operatorname{div} B_{h}=0 \quad \text { in } \Omega \times(0,+\infty), \\
D_{h}(0)=D_{0}, \quad B_{h}(0)=B_{0} \quad \text { in } \Omega, \\
D_{h} \times v=0, \quad B_{h} \cdot v=0 \quad \text { on } \Gamma \times(0,+\infty) .
\end{gathered}
$$

For our next purposes, we need that the following internal observability estimate holds: The subset $\omega$ of $\Omega$ is such that there exist a time $T>0$ and a constant $C>0$ such that

$$
\frac{1}{2} \int_{\Omega}\left(\lambda(x)\left|D_{0}(x)\right|^{2}+\mu(x)\left|B_{0}(x)\right|^{2}\right) d x \leq C \int_{0}^{T} \int_{\omega}\left|D_{h}(x, t)\right|^{2} d x d t, \quad \forall\left(D_{0}, B_{0}\right) \in H_{1},
$$

where

$$
H_{1}=\{(D, B) \in H: \operatorname{div} D=0 \text { in } \Omega\}
$$

This estimate was proved by Phung [17, Theorem 3.4] using microlocal analysis, when $\mu$ and $\lambda$ are constant and $\omega=\tilde{\omega} \cap \Omega$ such that $\tilde{\omega}$ controls geometrically $\Omega$. We will extend such an estimate to variable coefficients and some open subsets $\omega$ using the multiplier method. For that purpose, we further require that there exist $x_{0} \in \Omega$ and a positive constant $c_{0}$ such that

$$
\begin{aligned}
& \lambda(x)-\nabla \lambda(x) \cdot\left(x-x_{0}\right) \geq c_{0} \lambda(x), \\
& \mu(x)-\nabla \mu(x) \cdot\left(x-x_{0}\right) \geq c_{0} \mu(x)
\end{aligned}
$$

for all $x \in \Omega$.

We first reduce the estimate to the estimate of the electric field.

Lemma 3.1. Fix $T>0$. Let $\left(D_{h}, B_{h}\right)$ be the solution of (3.1), (3.2), (3.3), (3.4), and (3.5) with initial datum $\left(D_{0}, B_{0}\right) \in H_{1}$. Then there exists $C>0$ such that

$$
\frac{1}{2} \int_{\Omega}\left(\lambda(x)\left|D_{0}(x)\right|^{2}+\mu(x)\left|B_{0}(x)\right|^{2}\right) d x \leq C \int_{0}^{T} \int_{\Omega}\left|D_{h}(x, t)\right|^{2} d x d t, \quad \forall\left(D_{0}, B_{0}\right) \in H_{1} .
$$


Proof. We adapt step 1 of the proof of [17, Theorem 3.4] to our setting. Recall that the Hilbert space $H_{T}$ (curl, div, $\Omega$ ), defined in (2.15), equipped with its natural norm is compactly embedded into $\left(L^{2}(\Omega)\right)^{3}[20]$. Therefore there exists a unique $\psi \in H_{T}$ (curl, div, $\Omega$ ) solution of

$$
\begin{gathered}
\operatorname{curl}(\lambda \operatorname{curl} \psi)=B_{h} \quad \text { in } \Omega, \\
\operatorname{div} \psi=0 \quad \text { in } \Omega, \\
\psi \cdot \nu=0, \quad \operatorname{curl} \psi \times \nu=0 \quad \text { on } \Gamma,
\end{gathered}
$$

in the sense that $\psi \in H_{T}$ (curl, div, $\Omega$ ) is the unique solution of

$$
\int_{\Omega}\{\lambda \operatorname{curl} \psi \cdot \operatorname{curl} w+\operatorname{div} \psi \operatorname{div} w\} d x=\int_{\Omega} B_{h} \cdot w d x, \quad \forall w \in H_{T}(\operatorname{curl}, \operatorname{div}, \Omega) .
$$

Indeed the above compactness property and the hypotheses on $\Omega$ and $\Gamma$ guarantee that the left-hand side of (3.11) is coercive on $H_{T}(\operatorname{curl}, \operatorname{div}, \Omega)$. On the other hand since $\operatorname{div} B_{h}=0$ in $\Omega$ we easily see that the solution $\psi$ of (3.11) satisfies (3.10) (see [2, Theorem 1.1]). Setting $A=\operatorname{curl} \psi$, we deduce that

$$
\begin{gathered}
B_{h}=\operatorname{curl}(\lambda A) \quad \text { in } \Omega, \\
\operatorname{div} A=0 \quad \text { in } \Omega, \\
A \times \nu=0 \quad \text { on } \Gamma .
\end{gathered}
$$

Moreover taking $w=\psi$ in $(3.11)$ we see that

$$
\lambda_{0}\|A\|_{L^{2}(\Omega)^{3}}^{2} \leq\left\|B_{h}\right\|_{L^{2}(\Omega)^{3}}\|\psi\|_{L^{2}(\Omega)^{3}} \leq C\left\|B_{h}\right\|_{L^{2}(\Omega)^{3}}\|A\|_{L^{2}(\Omega)^{3}}
$$

this last estimate following from the compact embedding of $H_{T}$ (curl, div, $\Omega$ ) into $\left(L^{2}(\Omega)\right)^{3}$. In other words we have

$$
\|A\|_{L^{2}(\Omega)^{3}} \leq C\left\|B_{h}\right\|_{L^{2}(\Omega)^{3}}
$$

Using (3.2), (3.3), (3.5) and (3.12) to (3.14), we see that

$$
\begin{gathered}
\operatorname{curl}\left(\lambda\left(A^{\prime}+D_{h}\right)\right)=0 \quad \text { in } \Omega, \\
\operatorname{div}\left(A^{\prime}+D_{h}\right)=0 \quad \text { in } \Omega \\
\left(A^{\prime}+D_{h}\right) \times \nu=0 \quad \text { on } \Gamma .
\end{gathered}
$$

The first identity and the fact that $\Omega$ is simply connected imply that

$$
\lambda\left(A^{\prime}+D_{h}\right)=\nabla \varphi,
$$

with $\varphi \in H^{1}(\Omega)$. The properties (3.18), (3.19) and the fact that $\Gamma$ is connected imply that $\varphi$ is constant and therefore we conclude that

$$
A^{\prime}+D_{h}=0 \quad \text { in } \Omega
$$


798 Internal stabilization of Maxwell's equations

Take $\Phi(t)=t(T-t)$ and consider

$$
\int_{Q_{T}} \mu(x) \Phi(t)^{2}\left|B_{h}(x, t)\right|^{2} d x d t
$$

Then by (3.12) and Green's formula we get, owing to (3.14),

$$
\int_{Q_{T}} \mu(x) \Phi(t)^{2}\left|B_{h}(x, t)\right|^{2} d x d t=\int_{Q_{T}} \Phi(t)^{2} \operatorname{curl}\left(\mu B_{h}\right) \cdot \lambda A d x d t .
$$

Therefore by (3.1) we obtain

$$
\int_{Q_{T}} \mu(x) \Phi(t)^{2}\left|B_{h}(x, t)\right|^{2} d x d t=\int_{Q_{T}} \lambda \Phi(t)^{2} D_{h}^{\prime} \cdot A d x d t .
$$

Now by integration by parts in $t$, we get

$$
\int_{Q_{T}} \mu(x) \Phi(t)^{2}\left|B_{h}(x, t)\right|^{2} d x d t=-\int_{Q_{T}} \lambda\left(2 \Phi \Phi^{\prime} A+\Phi^{2} A^{\prime}\right) \cdot D_{h} d x d t
$$

The identity (3.21) then yields

$$
\int_{Q_{T}} \mu(x) \Phi(t)^{2}\left|B_{h}(x, t)\right|^{2} d x d t=-2 \int_{Q_{T}} \lambda \Phi \Phi^{\prime} A \cdot D_{h} d x d t+\int_{Q_{T}} \lambda \Phi^{2}\left|D_{h}\right|^{2} d x d t .
$$

Using Young's inequality we arrive at

$$
\begin{aligned}
\int_{Q_{T}} \mu(x) \Phi(t)^{2}\left|B_{h}(x, t)\right|^{2} d x d t \leq & \epsilon \int_{Q_{T}} \lambda \Phi^{2}|A|^{2} d x d t \\
& +\frac{1}{\epsilon} \int_{Q_{T}} \lambda\left(\Phi^{\prime}\right)^{2}\left|D_{h}\right|^{2} d x d t+\int_{Q_{T}} \lambda \Phi^{2}\left|D_{h}\right|^{2} d x d t
\end{aligned}
$$

for any $\epsilon>0$. Using finally the estimate (3.16) we have proved that

$$
\begin{aligned}
\int_{Q_{T}} \mu(x) \Phi(t)^{2}\left|B_{h}(x, t)\right|^{2} d x d t \leq & \frac{C \epsilon}{\mu_{0}} \int_{Q_{T}} \Phi^{2} \mu\left|B_{h}\right|^{2} d x d t \\
& +\frac{1}{\epsilon} \int_{Q_{T}} \lambda\left(\Phi^{\prime}\right)^{2}\left|D_{h}\right|^{2} d x d t+\int_{Q_{T}} \lambda \Phi^{2}\left|D_{h}\right|^{2} d x d t
\end{aligned}
$$

for any $\epsilon>0$. Choosing $\epsilon$ small enough we arrive at

$$
\int_{Q_{T}} \mu \Phi^{2}\left|B_{h}\right|^{2} d x d t \leq C \int_{Q_{T}} \lambda\left|D_{h}\right|^{2} d x d t .
$$

Using the conservation of energy (identity (2.28) with $\sigma=0$ ) we may write

$$
\int_{Q_{T}}\left(\mu\left|B_{h}\right|^{2}+\lambda\left|D_{h}\right|^{2}\right) d x d t=3 \int_{T / 3}^{2 T / 3} \int_{\Omega}\left(\mu\left|B_{h}\right|^{2}+\lambda\left|D_{h}\right|^{2}\right) d x d t .
$$


As $\Phi(t) \geq 2 T^{2} / 9$ on $[T / 3,2 T / 3]$ we get

$$
\int_{Q_{T}}\left(\mu\left|B_{h}\right|^{2}+\lambda\left|D_{h}\right|^{2}\right) d x d t \leq \frac{243}{4 T^{4}} \int_{T / 3}^{2 T / 3} \mu \Phi^{2}\left|B_{h}\right|^{2} d x d t+3 \int_{Q_{T}} \lambda\left|D_{h}\right|^{2} d x d t .
$$

The conclusion follows from (3.29).

Since it remains to estimate $\int_{0}^{T} \int_{\Omega}\left|D_{h}(x, t)\right|^{2} d x d t$ we are looking at $D_{h}$ as solution of the following second order system:

$$
\begin{gathered}
D_{h}^{\prime \prime}+\operatorname{curl}\left(\mu \operatorname{curl}\left(\lambda D_{h}\right)\right)=0 \quad \text { in } \Omega \times(0,+\infty), \\
\operatorname{div} D_{h}=0 \quad \text { in } \Omega \times(0,+\infty), \\
D_{h}(0)=D_{0}, \quad D_{h}^{\prime}(0)=D_{1}=\operatorname{curl}\left(\mu B_{0}\right) \quad \text { in } \Omega, \\
D_{h} \times \nu=0, \quad \operatorname{curl}\left(\lambda D_{h}\right) \cdot \nu=0 \quad \text { on } \Gamma \times(0,+\infty) .
\end{gathered}
$$

Consider the set

$$
\begin{aligned}
& H_{N}(\operatorname{curl}, \operatorname{div}, \Omega) \\
& \quad:=\left\{D \in L^{2}(\Omega)^{3}: \operatorname{curl} D \in L^{2}(\Omega)^{3}, \operatorname{div} D \in L^{2}(\Omega) ; D \times v=0 \text { on } \Gamma\right\},
\end{aligned}
$$

continuously embedded into $H^{1}(\Omega)^{3}$ (see, e.g., [5, Section I.3.4]) and compactly embedded into $L^{2}(\Omega)^{3}[20]$. Let us set

$$
\begin{aligned}
\mathscr{H} & :=\left\{D \in L^{2}(\Omega)^{3}: \operatorname{div} D=0 \text { in } \Omega\right\}, \\
\mathscr{V} & :=\left\{D \in H_{N}(\operatorname{curl}, \operatorname{div}, \Omega): \operatorname{div} D=0 \text { in } \Omega\right\}, \\
a\left(D, D_{1}\right) & :=\int_{\Omega} \mu \operatorname{curl}(\lambda D) \cdot \operatorname{curl}\left(\lambda D_{1}\right) d x, \quad \forall D, D_{1} \in \mathscr{V} .
\end{aligned}
$$

The bilinear form $a$ is symmetric and strongly coercive on $\mathscr{V}$, moreover $\mathscr{V}$ is compactly embedded into $\mathscr{H}$ (see [10]). By spectral analysis, the above problem has a unique solution $D_{h} \in C([0, T], \mathscr{V}) \cap C^{1}([0, T], \mathscr{H})$ if $\left(D_{0}, D_{1}\right)$ belongs to $\mathscr{V} \times \mathscr{H}$. Obviously $D_{h}$ is the same as the one from problem (3.1), (3.2), (3.3), (3.4), and (3.5) if $\left(D_{0}, B_{0}\right) \in \mathscr{V} \times$ $\left(\hat{J}(\Omega) \cap H^{1}(\Omega)^{3}\right)$, because then $\left(D_{0}, D_{1}=\operatorname{curl}\left(\mu B_{0}\right)\right)$ belongs to $\mathscr{V} \times \mathscr{H}$.

The energy of the solution of that system is given by

$$
E_{D}(t):=\frac{1}{2} \int_{\Omega}\left(\lambda(x)\left|D_{h}^{\prime}(x, t)\right|^{2}+\mu(x)\left|\operatorname{curl}\left(\lambda(x) D_{h}(x, t)\right)\right|^{2}\right) d x .
$$

A simple application of Green's formula shows that

$$
E_{D}^{\prime}(t)=0
$$

and therefore the energy $E_{D}$ is constant.

Using a vectorial multiplier method we first prove the following lemma. An analogous lemma was proved in [22] in the case of constant coefficients. 
800 Internal stabilization of Maxwell's equations

LemmA 3.2. Let $D_{h}$ be the solution of the system (3.32), (3.33), (3.34), and (3.35) with $\left(D_{0}, D_{1}\right) \in \mathscr{V} \times \mathscr{H}$, and let $q: \bar{\Omega} \rightarrow \mathbb{R}^{3}$ a $C^{1}$ vector field. Then for any time $T>0$ the following identity holds:

$$
\begin{aligned}
& {\left[\int_{\Omega} 2\left(D_{h}^{\prime}, q, \operatorname{curl}\left(\lambda D_{h}\right)\right) d x\right]_{0}^{T}} \\
& \quad=\int_{0}^{T} \int_{\Gamma}\left[\lambda(q \cdot v)\left|D_{h}^{\prime}\right|^{2}-\mu(q \cdot v)\left|\operatorname{curl}\left(\lambda D_{h}\right)\right|^{2}\right] d \Gamma d t \\
& \quad+\int_{0}^{T} \int_{\Omega}\left[\left(\lambda\left|D_{h}^{\prime}\right|^{2}+\mu\left|\operatorname{curl}\left(\lambda D_{h}\right)\right|^{2}\right) \operatorname{div} q-2 \lambda \sum_{i, j=1}^{3}\left(D_{h}^{\prime}\right)_{i}\left(D_{h}^{\prime}\right)_{j} \partial_{i} q_{j}\right. \\
& \left.\quad-2 \mu \sum_{i, j=1}^{3}\left(\operatorname{curl}\left(\lambda D_{h}\right)\right)_{i}\left(\operatorname{curl}\left(\lambda D_{h}\right)\right)_{j} \partial_{i} q_{j}\right] d x d t \\
& \quad-\int_{0}^{T} \int_{\Omega}\left[\left|D_{h}^{\prime}\right|^{2} q \cdot \nabla \lambda+\left|\operatorname{curl}\left(\lambda D_{h}\right)\right|^{2} q \cdot \nabla \mu\right] d x d t
\end{aligned}
$$

where the notation $(a, b, c)=a \cdot(b \times c)$ means the mixed product of the vectors $a, b, c$. Proof. By (3.32)

$$
\begin{aligned}
0= & \int_{0}^{T} \int_{\Omega} 2\left(D_{h}^{\prime \prime}+\operatorname{curl}\left(\mu \operatorname{curl}\left(\lambda D_{h}\right)\right), q, \operatorname{curl}\left(\lambda D_{h}\right)\right) d x d t \\
= & {\left[\int_{\Omega} 2\left(D_{h}^{\prime}, q, \operatorname{curl}\left(\lambda D_{h}\right)\right) d x\right]_{0}^{T}+\int_{0}^{T} \int_{\Gamma} 2 \mu\left(\nu, \operatorname{curl}\left(\lambda D_{h}\right), q \times \operatorname{curl}\left(\lambda D_{h}\right)\right) d \Gamma d t } \\
& +\int_{0}^{T} \int_{\Omega} 2\left[\mu \operatorname{curl}\left(\lambda D_{h}\right) \cdot \operatorname{curl}\left(q \times \operatorname{curl}\left(\lambda D_{h}\right)\right)-\left(D_{h}^{\prime}, q, \operatorname{curl}\left(\lambda D_{h}^{\prime}\right)\right)\right] d x d t
\end{aligned}
$$

Integrating by parts we obtain

$$
\begin{gathered}
\int_{0}^{T} \int_{\Omega}-2\left(D_{h}^{\prime}, q, \operatorname{curl}\left(\lambda D_{h}^{\prime}\right)\right) d x d t=\int_{0}^{T} \int_{\Omega} 2 \lambda D_{h}^{\prime} \cdot \operatorname{curl}\left(q \times D_{h}^{\prime}\right) d x d t \\
=\int_{0}^{T} \int_{\Omega} 2 \lambda\left[D_{h}^{\prime} \cdot\left(q \operatorname{div} D_{h}^{\prime}-D_{h}^{\prime} \operatorname{div} q\right)+\sum_{i, j=1}^{3}\left(D_{h}^{\prime}\right)_{i}\left(D_{h}^{\prime}\right)_{j} \partial_{i} q_{j}\right. \\
\left.\quad-\sum_{i, j=1}^{3}\left(D_{h}^{\prime}\right)_{j} q_{i} \partial_{i}\left(D_{h}^{\prime}\right)_{j}\right] d x d t \\
=\int_{0}^{T} \int_{\Omega}\left[2 \lambda \sum_{i, j=1}^{3}\left(D_{h}^{\prime}\right)_{i}\left(D_{h}^{\prime}\right)_{j} \partial_{i} q_{j}-2 \lambda\left|D_{h}^{\prime}\right|^{2} \operatorname{div} q-\lambda q \cdot \nabla\left(\left|D_{h}^{\prime}\right|^{2}\right)\right] d x d t
\end{gathered}
$$




$$
\begin{aligned}
= & \int_{0}^{T} \int_{\Omega}\left[2 \lambda \sum_{i, j=1}^{3}\left(D_{h}^{\prime}\right)_{i}\left(D_{h}^{\prime}\right)_{j} \partial_{i} q_{j}-2 \lambda\left|D_{h}^{\prime}\right|^{2} \operatorname{div} q+\left|D_{h}^{\prime}\right|^{2} \operatorname{div}(\lambda q)\right] d x d t \\
& -\int_{0}^{T} \int_{\Gamma} \lambda(q \cdot v)\left|D_{h}^{\prime}\right|^{2} d \Gamma d t,
\end{aligned}
$$

and then

$$
\begin{aligned}
\int_{0}^{T} \int_{\Omega} & -2\left(D_{h}^{\prime}, q, \operatorname{curl}\left(\lambda D_{h}^{\prime}\right)\right) d x d t \\
= & -\int_{0}^{T} \int_{\Gamma} \lambda(q \cdot v)\left|D_{h}^{\prime}\right|^{2} d \Gamma d t \\
& +\int_{0}^{T} \int_{\Omega}\left[2 \lambda \sum_{i, j=1}^{3}\left(D_{h}^{\prime}\right)_{i}\left(D_{h}^{\prime}\right)_{j} \partial_{i} q_{j}-\lambda\left|D_{h}^{\prime}\right|^{2} \operatorname{div} q+\left|D_{h}^{\prime}\right|^{2} q \cdot \nabla \lambda\right] d x d t .
\end{aligned}
$$

Analogously, we can rewrite

$$
\begin{gathered}
\int_{0}^{T} \int_{\Omega} 2 \mu \operatorname{curl}\left(\lambda D_{h}\right) \cdot \operatorname{curl}\left(q \times \operatorname{curl}\left(\lambda D_{h}\right)\right) d x d t \\
=\int_{0}^{T} \int_{\Omega} 2 \mu\left\{\operatorname{curl}\left(\lambda D_{h}\right) \cdot\left[q \operatorname{div} \operatorname{curl}\left(\lambda D_{h}\right)-\operatorname{curl}\left(\lambda D_{h}\right) \operatorname{div} q\right]\right. \\
\quad+\sum_{i, j=1}^{3}\left(\operatorname{curl}\left(\lambda D_{h}\right)\right)_{i}\left(\operatorname{curl}\left(\lambda D_{h}\right)\right)_{j} \partial_{i} q_{j} \\
\left.\quad-\sum_{i, j=1}^{3}\left(\operatorname{curl}\left(\lambda D_{h}\right)\right)_{j} q_{i} \partial_{i}\left(\operatorname{curl}\left(\lambda D_{h}\right)\right)_{j}\right\} d x d t \\
=\int_{0}^{T} \int_{\Omega}\left\{2 \mu\left[\sum_{i, j=1}^{3}\left(\operatorname{curl}\left(\lambda D_{h}\right)\right)_{i}\left(\operatorname{curl}\left(\lambda D_{h}\right)\right)_{j} \partial_{i} q_{j}-\left|\operatorname{curl}\left(\lambda D_{h}\right)\right|^{2} \operatorname{div} q\right]\right. \\
\left.\quad-\mu q \cdot \nabla\left(\left|\operatorname{curl}\left(\lambda D_{h}\right)\right|^{2}\right)\right\} d x d t \\
=\int_{0}^{T} \int_{\Omega}\left\{2 \mu\left[\sum_{i, j=1}^{3}\left(\operatorname{curl}\left(\lambda D_{h}\right)\right)_{i}\left(\operatorname{curl}\left(\lambda D_{h}\right)\right)_{j} \partial_{i} q_{j}-\left|\operatorname{curl}\left(\lambda D_{h}\right)\right|^{2} \operatorname{div} q\right]\right. \\
\left.\quad+\left|\operatorname{curl}\left(\lambda D_{h}\right)\right|^{2} \operatorname{div}(\mu q)\right\} d x d t-\int_{0}^{T} \int_{\Gamma} \mu(q \cdot v)\left|\operatorname{curl}\left(\lambda D_{h}\right)\right|^{2} d \Gamma d t,
\end{gathered}
$$


and then

$$
\begin{aligned}
& \int_{0}^{T} \int_{\Omega} 2 \mu \operatorname{curl}\left(\lambda D_{h}\right) \cdot \operatorname{curl}\left(q \times \operatorname{curl}\left(\lambda D_{h}\right)\right) d x d t \\
& =-\int_{0}^{T} \int_{\Gamma} \mu(q \cdot \nu)\left|\operatorname{curl}\left(\lambda D_{h}\right)\right|^{2} d \Gamma d t \\
& +\int_{0}^{T} \int_{\Omega}\left\{2 \mu\left[\sum_{i, j=1}^{3}\left(\operatorname{curl}\left(\lambda D_{h}\right)\right)_{i}\left(\operatorname{curl}\left(\lambda D_{h}\right)\right)_{j} \partial_{i} q_{j}-\mu\left|\operatorname{curl}\left(\lambda D_{h}\right)\right|^{2} \operatorname{div} q\right]\right. \\
& \left.+\left|\operatorname{curl}\left(\lambda D_{h}\right)\right|^{2} q \cdot \nabla \mu\right\} d x d t .
\end{aligned}
$$

Putting (3.43) and (3.45) in the first identity, we obtain

$$
\begin{gathered}
0=\left[\int_{\Omega} 2\left(D_{h}^{\prime}, q, \operatorname{curl}\left(\lambda D_{h}\right)\right) d x\right]_{0}^{T}+\int_{0}^{T} \int_{\Omega}\left[\left|D_{h}^{\prime}\right|^{2} q \cdot \nabla \lambda+\left|\operatorname{curl}\left(\lambda D_{h}\right)\right|^{2} q \cdot \nabla \mu\right] d x d t \\
+\int_{0}^{T} \int_{\Gamma}\left[2 \mu\left(\nu, \operatorname{curl}\left(\lambda D_{h}\right), q \times \operatorname{curl}\left(\lambda D_{h}\right)\right)-\lambda(q \cdot v)\left|D_{h}^{\prime}\right|^{2}\right. \\
\left.\quad-\mu(q \cdot v)\left|\operatorname{curl}\left(\lambda D_{h}\right)\right|^{2}\right] d \Gamma d t \\
+\int_{0}^{T} \int_{\Omega}\left[2 \lambda \sum_{i, j=1}^{3}\left(D_{h}^{\prime}\right)_{i}\left(D_{h}^{\prime}\right)_{j} \partial_{i} q_{j}+2 \mu \sum_{i, j=1}^{3}\left(\operatorname{curl}\left(\lambda D_{h}\right)\right)_{i}\left(\operatorname{curl}\left(\lambda D_{h}\right)\right)_{j} \partial_{i} q_{j}\right. \\
\left.-\left(\lambda\left|D_{h}^{\prime}\right|^{2}+\mu\left|\operatorname{curl}\left(\lambda D_{h}\right)\right|^{2}\right) \operatorname{div} q\right] d x d t .
\end{gathered}
$$

Therefore (3.40) follows observing that the boundary term can be rewritten using

$$
\begin{aligned}
2 \mu\left(\nu, \operatorname{curl}\left(\lambda D_{h}\right), q \times \operatorname{curl}\left(\lambda D_{h}\right)\right) \\
=2 \mu(q \cdot \nu)\left|\operatorname{curl}\left(\lambda D_{h}\right)\right|^{2} \\
\quad-2 \mu\left(\nu \cdot \operatorname{curl}\left(\lambda D_{h}\right)\right)\left(q \cdot \operatorname{curl}\left(\lambda D_{h}\right)\right) \\
=2 \mu(q \cdot v)\left|\operatorname{curl}\left(\lambda D_{h}\right)\right|^{2},
\end{aligned}
$$

recalling that $\operatorname{curl}\left(\lambda D_{h}\right) \cdot v=0$ on $\Gamma \times(0, \infty)$.

For any $\varepsilon>0$ let us denote by $\mathcal{N}_{\varepsilon}(\Gamma)$ the neighborhood of $\Gamma$ of radius $\varepsilon$, that is,

$$
\mathcal{N}_{\varepsilon}(\Gamma)=\left\{x \in \Omega: \inf _{y \in \Gamma}|x-y|<\varepsilon\right\} .
$$

Using the previous identity we prove the following lemma: 
Lemma 3.3. Let $D_{h}$ be the solution of the system (3.32), (3.33), (3.34), and (3.35) with $\left(D_{0}, D_{1}\right) \in \mathscr{V} \times \mathcal{H}$. If $\tilde{\omega}=\mathcal{N}_{\epsilon / 2}(\Gamma)$, for some $\epsilon>0$ and $\lambda$, $\mu$ satisfy (1.6), (3.8), then there exist $T_{0}>0$ and $C>0$ such that for $T>T_{0}$ we have

$$
\left(T-T_{0}\right) E_{D}(0) \leq C \int_{0}^{T} \int_{\tilde{\omega}}\left(\left|D_{h}^{\prime}(x, t)\right|^{2}+\left|D_{h}(x, t)\right|^{2}\right) d x d t .
$$

Proof. From (3.40), using the standard multiplier $q(x)=m(x)=x-x_{0}$, we obtain for any $T>0$

$$
\begin{aligned}
\int_{0}^{T} \int_{\Gamma}( & m \cdot v)\left[\lambda\left|D_{h}^{\prime}\right|^{2}-\mu\left|\operatorname{curl}\left(\lambda D_{h}\right)\right|^{2}\right] d \Gamma d t \\
= & {\left[\int_{\Omega} 2\left(D_{h}^{\prime}, m, \operatorname{curl}\left(\lambda D_{h}\right)\right) d x\right]_{0}^{T}-\int_{0}^{T} \int_{\Omega}\left[\lambda\left|D_{h}^{\prime}\right|^{2}+\mu\left|\operatorname{curl}\left(\lambda D_{h}\right)\right|^{2}\right] d x d t } \\
& +\int_{0}^{T} \int_{\Omega}\left[\left|D_{h}^{\prime}\right|^{2} m \cdot \nabla \lambda+\left|\operatorname{curl}\left(\lambda D_{h}\right)\right|^{2} m \cdot \nabla \mu\right] d x d t .
\end{aligned}
$$

Using the assumption (3.8), the above identity implies

$$
\begin{gathered}
c_{0} T \int_{\Omega}\left[\lambda\left|D_{1}\right|^{2}+\mu\left|\operatorname{curl}\left(\lambda D_{0}\right)\right|^{2}\right] d x-2\left[\int_{\Omega}\left(D_{h}^{\prime}, m, \operatorname{curl}\left(\lambda D_{h}\right)\right) d x\right]_{0}^{T} \\
\leq \int_{0}^{T} \int_{\Gamma}(m \cdot \nu)\left[\mu\left|\operatorname{curl}\left(\lambda D_{h}\right)\right|^{2}-\lambda\left|D_{h}^{\prime}\right|^{2}\right] d \Gamma d t
\end{gathered}
$$

Note that by (1.6)

$$
\left|\left[2 \int_{\Omega}\left(D_{h}^{\prime}, m, \operatorname{curl}\left(\lambda D_{h}\right)\right) d x\right]_{0}^{T}\right| \leq \frac{2 \max _{\bar{\Omega}}|m|}{\sqrt{\lambda_{0} \mu_{0}}} \int_{\Omega}\left[\lambda\left|D_{1}\right|^{2}+\mu\left|\operatorname{curl}\left(\lambda D_{0}\right)\right|^{2}\right] d x .
$$

So, setting

$$
\tilde{T}=\frac{2 \max _{\bar{\Omega}}|m|}{c_{0} \sqrt{\lambda_{0} \mu_{0}}}
$$

we obtain

$$
\begin{aligned}
c_{0}(T & -\tilde{T}) \int_{\Omega}\left(\lambda\left|D_{1}\right|^{2}+\mu\left|\operatorname{curl}\left(\lambda D_{0}\right)\right|^{2}\right) d x \\
& \leq \int_{0}^{T} \int_{\Gamma}(m \cdot \nu)\left[\mu\left|\operatorname{curl}\left(\lambda D_{h}\right)\right|^{2}-\lambda\left|D_{h}^{\prime}\right|^{2}\right] d \Gamma d t .
\end{aligned}
$$

Now, set $\omega_{0}=\mathcal{N}_{\varepsilon / 4}(\Gamma)$ and apply (3.40) using as multiplier $q(x)=\varphi(x) m(x)$ with $\varphi \in$ $C^{1}(\bar{\Omega}), 0 \leq \varphi(x) \leq 1$,

$$
\varphi(x) \equiv 1, \quad x \in \mathcal{N}_{\varepsilon / 8}(\Gamma), \quad \varphi(x) \equiv 0, \quad x \in \Omega \backslash \omega_{0} .
$$


804 Internal stabilization of Maxwell's equations

We obtain

$$
\begin{aligned}
\int_{0}^{T} \int_{\Gamma}(m \cdot v)\left[\mu\left|\operatorname{curl}\left(\lambda D_{h}\right)\right|^{2}-\lambda\left|D_{h}^{\prime}\right|^{2}\right] d \Gamma d t \\
\leq C \int_{0}^{T} \int_{\omega_{0}}\left(\left|D_{h}^{\prime}\right|^{2}+\left|\operatorname{curl}\left(\lambda D_{h}\right)\right|^{2}\right) d x d t \\
\quad+c_{0} \tilde{T} \int_{\Omega}\left(\lambda\left|D_{1}\right|^{2}+\mu\left|\operatorname{curl}\left(\lambda D_{0}\right)\right|^{2}\right) d x
\end{aligned}
$$

for a suitable constant $C>0$. Then, from (3.54) and (3.56),

$$
c_{0}(T-2 \tilde{T}) \int_{\Omega}\left(\lambda\left|D_{1}\right|^{2}+\mu\left|\operatorname{curl}\left(\lambda D_{0}\right)\right|^{2}\right) d x \leq C \int_{0}^{T} \int_{\omega_{0}}\left(\left|D_{h}^{\prime}\right|^{2}+\left|\operatorname{curl}\left(\lambda D_{h}\right)\right|^{2}\right) d x d t
$$

Now, let $g: \bar{\Omega} \rightarrow \mathbb{R}$ be a $C^{1}$ function with $0 \leq g(x) \leq 1$, and

$$
g(x) \equiv 1, \quad x \in \omega_{0}, \quad g(x) \equiv 0, \quad x \in \Omega \backslash \tilde{\omega} .
$$

By (3.32), for any positive time $\mathrm{T}$, by integration by parts, we have

$$
\begin{aligned}
0= & \int_{0}^{T} \int_{\Omega}\left[D_{h}^{\prime \prime}+\operatorname{curl}\left(\mu \operatorname{curl}\left(\lambda D_{h}\right)\right)\right] \cdot\left(g \lambda D_{h}\right) d x d t=\left[\int_{\Omega} \lambda g D_{h}^{\prime} \cdot D_{h} d x\right]_{0}^{T} \\
& -\int_{0}^{T} \int_{\Omega} \lambda g\left|D_{h}^{\prime}\right|^{2} d x d t+\int_{0}^{T} \int_{\Omega} \mu \operatorname{curl}\left(\lambda D_{h}\right) \cdot\left[-\lambda D_{h} \times \nabla g+g \operatorname{curl}\left(\lambda D_{h}\right)\right] d x d t .
\end{aligned}
$$

Then,

$$
\begin{aligned}
\int_{0}^{T} \int_{\Omega} \mu g\left|\operatorname{curl}\left(\lambda D_{h}\right)\right|^{2} d x d t= & \int_{0}^{T} \int_{\Omega} \lambda g\left|D_{h}^{\prime}\right|^{2} d x d t-\left[\int_{\Omega} \lambda g D_{h}^{\prime} \cdot D_{h} d x\right]_{0}^{T} \\
& +2 \int_{0}^{T} \int_{\Omega} \mu \sqrt{g} \operatorname{curl}\left(\lambda D_{h}\right) \cdot\left(\lambda D_{h} \times \nabla \sqrt{g}\right) d x d t .
\end{aligned}
$$

By Young's inequality we can estimate

$$
\begin{aligned}
& \left|2 \int_{0}^{T} \int_{\Omega} \mu \sqrt{g} \operatorname{curl}\left(\lambda D_{h}\right) \cdot\left(\lambda D_{h} \times \nabla \sqrt{g}\right) d x d t\right| \\
& \quad \leq \frac{1}{2} \int_{0}^{T} \int_{\tilde{\omega}} \mu g\left|\operatorname{curl}\left(\lambda D_{h}\right)\right|^{2} d x d t+C \int_{0}^{T} \int_{\tilde{\omega}}\left|D_{h}\right|^{2} d x d t .
\end{aligned}
$$


Moreover, using the inequality

$$
\int_{\Omega}\left|D_{h}\right|^{2} d x \leq C \int_{\Omega}\left|\operatorname{curl}\left(\lambda D_{h}\right)\right|^{2} d x
$$

consequence of the compact embedding of $H_{N}(\operatorname{curl}, \operatorname{div}, \Omega)$ into $L^{2}(\Omega)^{3}$, we have

$$
\left|\left[\int_{\Omega} \lambda g D_{h}^{\prime} \cdot D_{h} d x\right]_{0}^{T}\right| \leq C \int_{\Omega}\left(\lambda\left|D_{h}^{\prime}\right|^{2}+\mu\left|\operatorname{curl}\left(\lambda D_{h}\right)\right|^{2}\right) d x
$$

Therefore, using (3.61) and (3.63) in (3.60), we obtain

$$
\begin{aligned}
& \int_{0}^{T} \int_{\omega_{0}}\left|\operatorname{curl}\left(\lambda D_{h}\right)\right|^{2} d x d t \leq \int_{0}^{T} \int_{\tilde{\omega}} g\left|\operatorname{curl}\left(\lambda D_{h}\right)\right|^{2} d x d t \\
& \quad \leq C \int_{\Omega}\left(\lambda\left|D_{h}^{\prime}\right|^{2}+\mu\left|\operatorname{curl}\left(\lambda D_{h}\right)\right|^{2}\right) d x+C^{\prime} \int_{0}^{T} \int_{\tilde{\omega}}\left(\left|D_{h}\right|^{2}+\left|D_{h}^{\prime}\right|^{2}\right) d x d t
\end{aligned}
$$

for suitable positive constants $C, C^{\prime}$. Finally, by (3.57) and (3.64) we have

$$
(T-2 \tilde{T}) E_{D}(0) \leq C \int_{0}^{T} \int_{\tilde{\omega}}\left(\left|D_{h}^{\prime}\right|^{2}+\left|D_{h}\right|^{2}\right) d x d t+C E_{D}(0)
$$

for some constant $C>0$. So, we can deduce the existence of a time $T_{0}$ such that for $T>T_{0}$

$$
\left(T-T_{0}\right) E_{D}(0) \leq \int_{0}^{T} \int_{\tilde{\omega}}\left(\left|D_{h}^{\prime}\right|^{2}+\left|D_{h}\right|^{2}\right) d x d t
$$

In a second step using a duality argument as in [1] (see also [12, Lemma 10]) we prove the following estimate.

Lemma 3.4. Let $D_{h}$ be the solution of the system (3.32), (3.33), (3.34), and (3.35) with $\left(D_{0}, D_{1}\right) \in \mathscr{V} \times \mathscr{H}$. If $\omega=\mathcal{N}_{\epsilon}(\Gamma)$ and $\tilde{\omega}=\mathcal{N}_{\epsilon / 2}(\Gamma)$, for some $\epsilon>0$, then there exists $C>0$ such that for any $\eta>0$ we have

$$
\int_{0}^{T} \int_{\tilde{\omega}}\left|D_{h}(x, t)\right|^{2} d x d t \leq \frac{C}{\eta} \int_{0}^{T} \int_{\omega}\left|D_{h}^{\prime}(x, t)\right|^{2} d x d t+\eta \int_{0}^{T} E_{D}(t) d t+C E_{D}(0) .
$$


Proof. Fix $\beta \in \mathscr{D}\left(\mathbb{R}^{3}\right)$ such that $\beta \equiv 1$ on $\tilde{\omega}$ with a support included into $\omega$.

Consider $z \in H_{N}(\operatorname{curl}, \operatorname{div}, \Omega)$ the unique solution of

$$
\int_{\Omega} \mu \operatorname{curl}(\lambda z) \cdot \operatorname{curl}(\lambda w) d x+\int_{\Omega} \operatorname{div} z \operatorname{div} w d x=\int_{\Omega} \beta \lambda D_{h}(x, t) \cdot w(x) d x,
$$

for all $w \in H_{N}$ (curl, div, $\Omega$ ). This solution $z$ satisfies (due to the compact embedding of $H_{N}(\operatorname{curl}, \operatorname{div}, \Omega)$ in $L^{2}(\Omega)^{3}$ and to the properties of $\Omega$ and $\Gamma$ )

$$
\|z\|_{L^{2}(\Omega)^{3}} \leq C\left\|\beta D_{h}\right\|_{L^{2}(\Omega)^{3}},
$$

for some $C>0$.

Multiplying (3.32) by $\lambda z$ and integrating in $Q_{T}$ we get

$$
0=\int_{Q_{T}} \lambda\left(D_{h}^{\prime \prime}+\operatorname{curl}\left(\mu \operatorname{curl}\left(\lambda D_{h}\right)\right)\right) \cdot z d x d t
$$

Applying Green's formula (in space and time) and taking into account the boundary condition $z \times v=0$ on $\Gamma$ we obtain

$$
0=-\int_{Q_{T}} \lambda D_{h}^{\prime} z^{\prime} d x d t+\left[\int_{\Omega} \lambda D_{h}^{\prime} z d x\right]_{0}^{T}+\int_{Q_{T}} \mu \operatorname{curl}\left(\lambda D_{h}\right) \cdot \operatorname{curl}(\lambda z) d x d t .
$$

Now taking into account (3.33) and using (3.68) with $w=D_{h}$ we arrive at

$$
0=-\int_{Q_{T}} \lambda D_{h}^{\prime} z^{\prime} d x d t+\left[\int_{\Omega} \lambda D_{h}^{\prime} z d x\right]_{0}^{T}+\int_{Q_{T}} \beta \lambda\left|D_{h}\right|^{2} d x d t
$$

By Cauchy-Schwarz's inequality and the fact that $\beta \equiv 1$ on $\tilde{\omega}$, we get

$$
\begin{aligned}
\int_{0}^{T} \int_{\tilde{\omega}} \lambda\left|D_{h}\right|^{2} d x d t & \leq \int_{Q_{T}} \beta \lambda\left|D_{h}\right|^{2} d x d t=\int_{Q_{T}} \lambda D_{h}^{\prime} z^{\prime} d x d t-\left[\int_{\Omega} \lambda D_{h}^{\prime} z d x\right]_{0}^{T} \\
& \leq\left(\int_{Q_{T}} \lambda\left|D_{h}^{\prime}\right|^{2} d x d t\right)^{1 / 2}\left(\int_{Q_{T}} \lambda\left|z^{\prime}\right|^{2} d x d t\right)^{1 / 2} \\
& +\left.\left(\int_{\Omega} \lambda\left|D_{h}^{\prime}(x, t)\right|^{2} d x\right)^{1 / 2}\left(\int_{\Omega} \lambda|z(x, t)|^{2} d x\right)^{1 / 2}\right|_{t=0, T}
\end{aligned}
$$


Using the estimates (3.69), (3.62) and the definition of the energy we get

$$
\begin{aligned}
\int_{0}^{T} \int_{\tilde{\omega}} \lambda\left|D_{h}\right|^{2} d x d t & \leq C\left(\int_{Q_{T}} \lambda\left|D_{h}^{\prime}\right|^{2} d x d t\right)^{1 / 2}\left(\int_{Q_{T}} \beta\left|D_{h}^{\prime}\right|^{2} d x d t\right)^{1 / 2}+C E_{D}(0) \\
& \leq C\left(\int_{0}^{T} E_{D}(t) d t\right)^{1 / 2}\left(\int_{0}^{T} \int_{\omega}\left|D_{h}^{\prime}\right|^{2} d x d t\right)^{1 / 2}+C E_{D}(0)
\end{aligned}
$$

We conclude by Young's inequality.

Corollary 3.5. Let $D_{h}$ be the solution of the system (3.32), (3.33), (3.34), and (3.35) with $\left(D_{0}, D_{1}\right) \in \mathscr{V} \times \mathscr{H}$. If $\omega=\mathcal{N}_{\epsilon}(\Gamma)$, for some $\epsilon>0$ and $\lambda$, $\mu$ satisfy (1.6), (3.8), then there exist $T_{1}>0$ and $C>0$ such that for $T>T_{1}$ we have

$$
\left(T-T_{1}\right) E_{D}(0) \leq C \int_{0}^{T} \int_{\omega}\left|D_{h}^{\prime}(x, t)\right|^{2} d x d t .
$$

Proof. By (3.49) and (3.67) we may write

$$
\begin{aligned}
\left(T-T_{0}\right) E_{D}(0) \leq & C \int_{0}^{T} \int_{\tilde{\omega}}\left|D_{h}^{\prime}(x, t)\right|^{2} d x d t \\
& +\frac{C}{\eta} \int_{0}^{T} \int_{\omega}\left|D_{h}^{\prime}(x, t)\right|^{2} d x d t+C \eta \int_{0}^{T} E_{D}(t) d t+C E_{D}(0),
\end{aligned}
$$

for any $\eta>0$. By the conservation of energy, this yields

$$
\left(T-T_{0}\right) E_{D}(0) \leq C \int_{0}^{T} \int_{\tilde{\omega}}\left|D_{h}^{\prime}(x, t)\right|^{2} d x d t+\frac{C}{\eta} \int_{0}^{T} \int_{\omega}\left|D_{h}^{\prime}(x, t)\right|^{2} d x d t+C(\eta T+1) E_{D}(0) .
$$

The conclusion follows by choosing $\eta$ small enough.

We now finish by adapting a weakening of norm argument from [11, Section VII.2.4]. Lemma 3.6. Fix $T>T_{1}$. Let $\left(D_{h}, B_{h}\right)$ be the solution of (3.1), (3.2), (3.3), (3.4), and (3.5) with initial datum $\left(D_{0}, B_{0}\right) \in H_{1}$. If $\omega=\mathcal{N}_{\epsilon}(\Gamma)$, for some $\epsilon>0$, then there exists $C>0$ (depending on $T$ ) such that

$$
\int_{0}^{T} \int_{\Omega}\left|D_{h}(x, t)\right|^{2} d x d t \leq C \int_{0}^{T} \int_{\omega}\left|D_{h}(x, t)\right|^{2} d x d t
$$


808 Internal stabilization of Maxwell's equations

Proof. We only need to prove (3.78) for $\left(D_{0}, B_{0}\right) \in \mathscr{V} \times\left(\hat{J}(\Omega) \cap H^{1}(\Omega)^{3}\right)$ since this space is dense in $H_{1}([9,10])$.

Consider $\chi \in H_{N}(\operatorname{curl}, \operatorname{div}, \Omega)$ the unique solution of $\left(\right.$ with $\left.D_{1}=\operatorname{curl}\left(\mu B_{0}\right)\right)$

$$
\begin{gathered}
\operatorname{curl}(\mu \operatorname{curl}(\lambda \chi))=D_{1} \quad \text { in } \Omega, \\
\operatorname{div} \chi=0 \quad \text { in } \Omega, \\
\chi \times \nu=0, \quad \operatorname{curl}(\lambda \chi) \cdot \nu=0 \quad \text { on } \Gamma,
\end{gathered}
$$

in the sense that $\chi \in H_{N}(\operatorname{curl}, \operatorname{div}, \Omega)$ is the unique solution of

$$
\int_{\Omega}\{\mu \operatorname{curl}(\lambda \chi) \cdot \operatorname{curl}(\lambda w)+\operatorname{div} \chi \operatorname{div} w\} d x=\int_{\Omega} \lambda D_{1} \cdot w d x, \quad \forall w \in H_{N}(\operatorname{curl}, \operatorname{div}, \Omega) .
$$

Set

$$
w(t)=\int_{0}^{t} D_{h}(s) d s+\chi
$$

Then from (3.32), (3.33), (3.34), and (3.35) and (3.79), we see that $w$ satisfies (3.32), (3.33), (3.35) and the initial conditions

$$
w(0)=\chi \in \mathscr{V}, \quad w^{\prime}(0)=D_{0} \in \mathscr{H} .
$$

Therefore by Corollary 3.5 we have

$$
\frac{T-T_{1}}{2 T} \int_{0}^{T} \int_{\Omega}\left(\lambda(x)\left|w^{\prime}(x, t)\right|^{2}+\mu(x)|\operatorname{curl}(\lambda w(x, t))|^{2}\right) d x d t \leq C \int_{0}^{T} \int_{\omega}\left|w^{\prime}(x, t)\right|^{2} d x d t .
$$

This estimate directly leads to the conclusion as $w^{\prime}=D_{h}$.

By Lemmas 3.1 and 3.6 we directly conclude the following theorem.

Theorem 3.7. If $\omega=\mathcal{N}_{\epsilon}(\Gamma)$, for some $\epsilon>0$, and $\lambda$, $\mu$ satisfy (1.6), (3.8), then (3.6) holds for T large enough.

\section{The stability result}

Based on the stability estimate of the previous section, we deduce our main result.

Theorem 4.1. Let $\omega$ be a subset of $\Omega$ such that (3.6) holds. Assume that $\sigma$ satisfies (1.7). Then there exist $C \geq 1$ and $\gamma>0$ such that

$$
\mathscr{E}(t) \leq C e^{-\gamma t \mathscr{E}(0),}
$$

for every solution $(D, B)$ of the system (1.1), (1.2), (1.3), (1.4), and (1.5) with initial datum in $\mathrm{H}_{1}$. 
Proof. As in [24, Theorem 1.1], we split up $(D, B)$, solution of $(1.1),(1.2),(1.3),(1.4)$, and (1.5) as follows:

$$
(D, B)=\left(D_{h}, B_{h}\right)+\left(D_{n h}, B_{n h}\right)
$$

where $\left(D_{h}, B_{h}\right)$ is solution of (3.1), (3.2), (3.3), (3.4), and (3.5) and $\left(D_{n h}, B_{n h}\right)$ is the remainder which then satisfies

$$
\begin{gathered}
D_{n h}^{\prime}-\operatorname{curl}\left(\mu B_{n h}\right)=-\sigma D \quad \text { in } \Omega \times(0,+\infty), \\
B_{n h}^{\prime}+\operatorname{curl}\left(\lambda D_{n h}\right)=0 \quad \text { in } \Omega \times(0,+\infty), \\
\operatorname{div} B_{n h}=0 \quad \text { in } \Omega \times(0,+\infty), \\
D_{n h}(0)=0, \quad B_{n h}(0)=0 \quad \text { in } \Omega, \\
D_{n h} \times v=0, \quad B_{n h} \cdot v=0 \quad \text { on } \Gamma \times(0,+\infty) .
\end{gathered}
$$

Equivalently $\left(D_{n h}, B_{n h}\right)$ satisfies (2.16), (2.17), (2.18), (2.19), and (2.20) with $f=-\sigma D$. Therefore by Theorem 2.3, it holds

$$
\int_{Q_{T}}\left\{\left|D_{n h}(x, t)\right|^{2}+\left|B_{n h}(x, t)\right|^{2}\right\} d x d t \leq C T^{2} \int_{Q_{T}}|\sigma D(x, t)|^{2} d x d t
$$

and since $\sigma$ is bounded we get

$$
\int_{Q_{T}}\left\{\left|D_{n h}(x, t)\right|^{2}+\left|B_{n h}(x, t)\right|^{2}\right\} d x d t \leq C T^{2} \max _{x \in \bar{\Omega}} \sigma(x) \int_{Q_{T}} \sigma|D(x, t)|^{2} d x d t .
$$

On the other hand by (3.6) we have

$$
\begin{aligned}
\mathscr{E}(T) & \leq \mathscr{E}(0)=\frac{1}{2} \int_{\Omega}\left(\lambda(x)\left|D_{0}(x)\right|^{2}+\mu(x)\left|B_{0}(x)\right|^{2}\right) d x \\
& \leq C \int_{0}^{T} \int_{\omega}\left|D_{h}(x, t)\right|^{2} d x d t \\
& \leq C \int_{0}^{T} \int_{\omega}\left\{|D(x, t)|^{2}+\left|D_{n h}(x, t)\right|^{2}\right\} d x d t \\
& \leq \frac{C}{\sigma_{0}} \int_{0}^{T} \int_{\omega} \sigma|D(x, t)|^{2} d x d t+C \int_{0}^{T} \int_{\omega}\left|D_{n h}(x, t)\right|^{2} d x d t .
\end{aligned}
$$

By (4.5) we conclude that

$$
\mathscr{E}(T) \leq C \int_{Q_{T}} \sigma|D(x, t)|^{2} d x d t
$$

which leads to the conclusion due to (2.25), using a standard argument (see, e.g., [3, Theoremm 3.3] or [14, Section 3]). 


\section{References}

[1] F. Conrad and B. Rao, Decay of solutions of the wave equation in a star-shaped domain with nonlinear boundary feedback, Asymptotic Anal. 7 (1993), no. 3, 159-177.

[2] M. Costabel, M. Dauge, and S. Nicaise, Singularities of Maxwell interface problems, M2AN Math. Model. Numer. Anal. 33 (1999), no. 3, 627-649.

[3] M. Eller, J. E. Lagnese, and S. Nicaise, Stabilization of heterogeneous Maxwell's equations by linear or nonlinear boundary feedback, Electron. J. Differential Equations 2002 (2002), no. 21, 126.

[4] M. M. Eller and J. E. Masters, Exact boundary controllability of electromagnetic fields in a general region, Appl. Math. Optim. 45 (2002), no. 1, 99-123.

[5] V. Girault and P.-A. Raviart, Finite Element Methods for Navier-Stokes Equations. Theory and Algorithms, Springer Series in Computational Mathematics, vol. 5, Springer, Berlin, 1986.

[6] B. V. Kapitonov, Stabilization and exact boundary controllability for Maxwell's equations, SIAM J. Control Optim. 32 (1994), no. 2, 408-420.

[7] K. A. Kime, Boundary controllability of Maxwell's equations in a spherical region, SIAM J. Control Optim. 28 (1990), no. 2, 294-319.

[8] V. Komornik, Boundary stabilization, observation and control of Maxwell's equations, Panamer. Math. J. 4 (1994), no. 4, 47-61.

[9] O. A. Ladyzhenskaya and V. A. Solonnikov, The linearization principle and invariant manifolds for problems of magnetohydrodynamics, J. Soviet Math. 8 (1977), 384-422.

[10] J. E. Lagnese, Exact boundary controllability of Maxwell's equations in a general region, SIAM J. Control Optim. 27 (1989), no. 2, 374-388.

[11] J.-L. Lions, Contrôlabilité exacte, perturbations et stabilisation de systèmes distribués. Tome 1. [Exact controllability, perturbations and stabilization of distributed systems. Vol. 1], Recherches en Mathématiques Appliquées [Research in Applied Mathematics], vol. 8, Masson, Paris, 1988.

[12] P. Martinez, A new method to obtain decay rate estimates for dissipative systems with localized damping, Rev. Mat. Complut. 12 (1999), no. 1, 251-283.

[13] S. Nicaise, Exact boundary controllability of Maxwell's equations in heterogeneous media and an application to an inverse source problem, SIAM J. Control Optim. 38 (2000), no. 4, 11451170.

[14] Stability and controllability of an abstract evolution equation of hyperbolic type and concrete applications, Rend. Mat. Appl. (7) 23 (2003), no. 1, 83-116.

[15] S. Nicaise and C. Pignotti, Boundary stabilization of Maxwell's equations with space-time variable coefficients, ESAIM Control Optim. Calc. Var. 9 (2003), 563-578.

[16] A. Pazy, Semigroups of Linear Operators and Applications to Partial Differential Equations, Applied Mathematical Sciences, vol. 44, Springer, Berlin, 1983.

[17] K. D. Phung, Contrôle et stabilisation d'ondes électromagnétiques [Control and stabilization of electromagnetic waves], ESAIM Control Optim. Calc. Var. 5 (2000), 87-137 (French).

[18] C. Pignotti, Observability and controllability of Maxwell's equations, Rend. Mat. Appl. (7) 19 (1999), no. 4, 523-546.

[19] D. L. Russell, The Dirichlet-Neumann boundary control problem associated with Maxwell's equations in a cylindrical region, SIAM J. Control Optim. 24 (1986), no. 2, 199-229.

[20] Ch. Weber, A local compactness theorem for Maxwell's equations, Math. Methods Appl. Sci. 2 (1980), no. 1, 12-25.

[21] N. Weck, Exact boundary controllability of a Maxwell problem, SIAM J. Control Optim. 38 (2000), no. 3, 736-750.

[22] X. Zhang, Exact internal controllability of Maxwell's equations, Appl. Math. Optim. 41 (2000), no. $2,155-170$. 
[23] Q. Zhou, Exact internal controllability of Maxwell's equations, Japan J. Indust. Appl. Math. 14 (1997), no. 2, 245-256.

[24] E. Zuazua, Exponential decay for the semilinear wave equation with locally distributed damping, Comm. Partial Differential Equations 15 (1990), no. 2, 205-235.

Serge Nicaise: MACS, Institut des Sciences et Techniques de Valenciennes, Université de Valenciennes et du Hainaut Cambrésis, 59313 Valenciennes Cedex 9, France

E-mail address: snicaise@univ-valenciennes.fr

Cristina Pignotti: Dipartimento di Matematica Pura e Applicata, Università di L'Aquila Via Vetoio, Loc. Coppito, 67010 L’Aquila, Italy

E-mail address: pignotti@univaq.it 


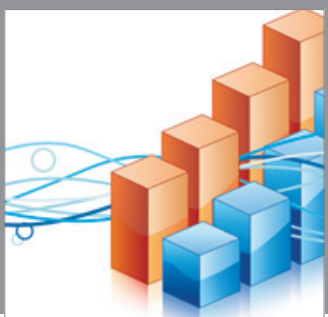

Advances in

Operations Research

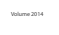

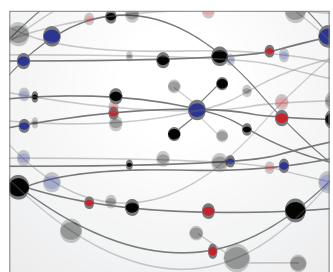

\section{The Scientific} World Journal
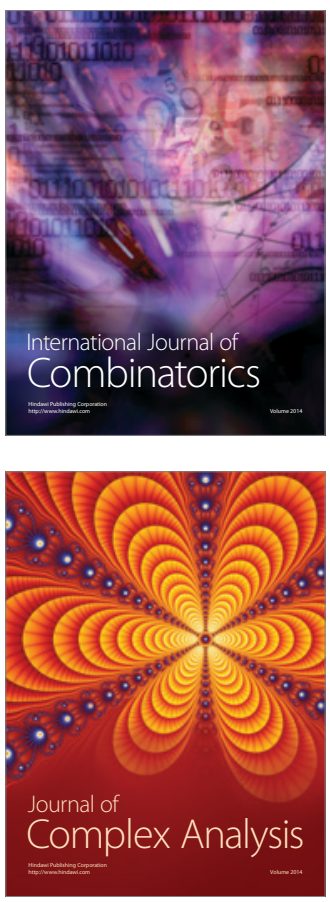

International Journal of

Mathematics and

Mathematical

Sciences
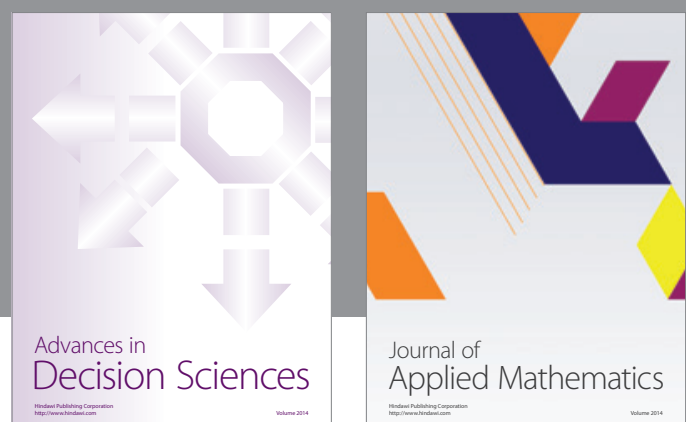

Journal of

Applied Mathematics
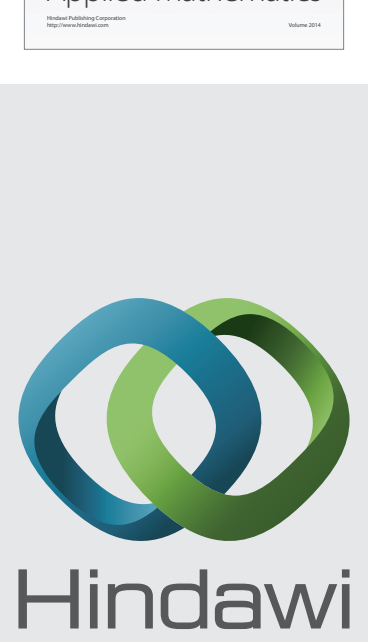

Submit your manuscripts at http://www.hindawi.com
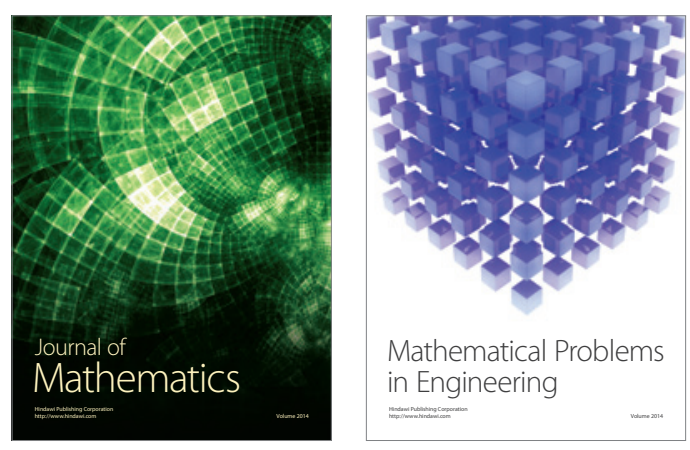

Mathematical Problems in Engineering
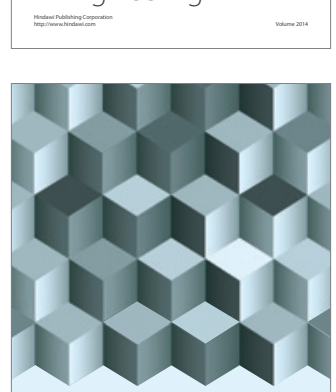

Journal of

Function Spaces
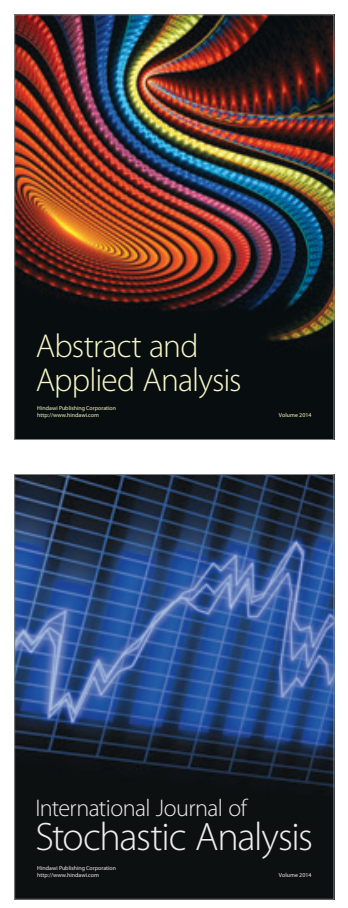

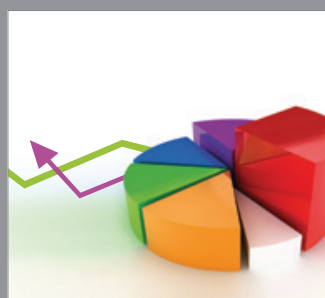

ournal of

Probability and Statistics

Promensencen
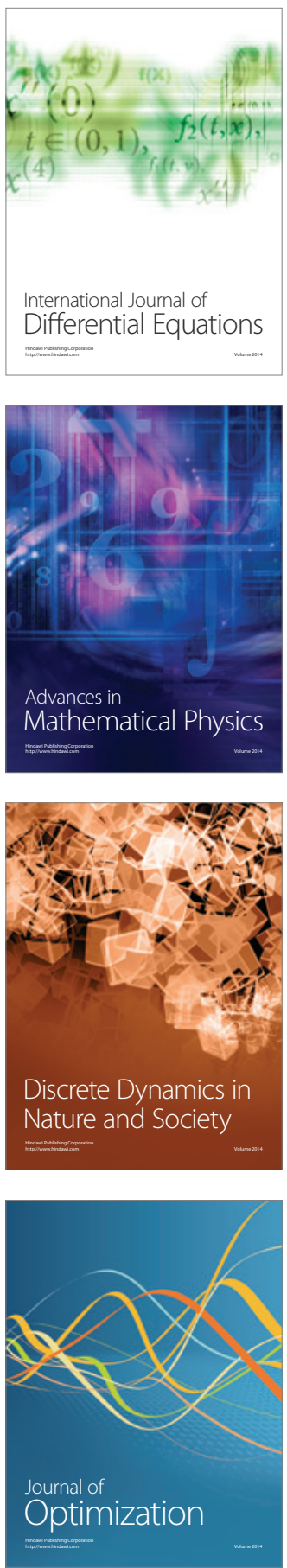